\title{
Anabases
}

ANABASES Traditions et réceptions de l'Antiquité

12 | 2010

Varia

\section{Occidere pro patria : quelques réflexions sur le patriotisme}

\section{Claudia Moatti}

\section{(2) OpenEdition}

\section{Journals}

Édition électronique

URL : http://journals.openedition.org/anabases/1192

DOI : 10.4000/anabases. 1192

ISSN : 2256-9421

Éditeur

E.R.A.S.M.E.

Édition imprimée

Date de publication : 1 octobre 2010

Pagination : 137-147

ISSN : 1774-4296

\section{Référence électronique}

Claudia Moatti, «Occidere pro patria : quelques réflexions sur le patriotisme », Anabases [En ligne], 12

2010, mis en ligne le 01 octobre 2013, consulté le 20 octobre 2019. URL : http://

journals.openedition.org/anabases/1192 ; DOI : 10.4000/anabases.1192 


\section{Occidere pro patria : quelques réflexions sur le patriotisme}

Claudia Moatti

" Il est sûr que ma patrie n'est pas là où les actes les plus infamants sont quotidiens et où chaque heure voit se préparer l'abomination des abominations. " Klaus Mann, Contre la barbarie, 1925-1948, trad. D.L. Miermont et C. Gepner, Paris, 2010, p. 99-100.

L' AMOR PATRIAE ET L'HUMANITAS définissent deux devoirs, l'un civique, se battre pour la patrie, et l'autre moral, respecter l'homme en l'homme, qui relèvent tous deux de ce que l'on pourrait appeler le souci des autres, mais qui sont en tension. Non pas tant si l'on ne voit du patriotisme que le sacrifice de soi - mori pro patria, dont les Anciens avaient souligné la nature "rédemptrice ", comme ils avaient analysé la joie qui résulte de ce profond accord du citoyen avec son devoir pour les siens ${ }^{1}-$; mais si l'on met

1 Sénèque, ad Lucilium 9, 76, 26-27 : "J'ai posé en principe qu'on doit tout braver pour l'honnête ; ce qui serait une folie, s'il existait d'autre bien [...]. Mais tu ne comprendras jamais ce sentiment [...] à moins de te poser cette question : "Si les circonstances exigeaient que je mourusse pour la patrie et qu'il fallût racheter la vie de mes concitoyens au prix de la mienne, présenterais-je ma tête, non-seulement avec courage, mais encore avec plaisir ?" Si tu es capable de faire pareille chose, c'est qu'à tes yeux il n'est pas d'autre bien, puisque tu quittes tout pour le posséder. Vois le pouvoir de la vertu ! Tu mourras pour la patrie, dusses-tu le faire sur-le-champ, du moment que tu seras convaincu qu'il faut le faire. " (Numquam autem vera tibi opinio talis videbitur, nisi animum adleves et te ipse interroges, si res exegerit ut pro patria moriaris et salutem omnium civium tua redimas, an porrecturus sis cervicem non tantum patienter sed etiam libenter. Si hoc facturus es, nullum 
l'accent sur le sacrifice de l'autre, sur la nécessité de tuer pour la patrie - occidere pro patria. Le patriotisme présente en effet deux faces, tout comme la devotio latine, l'acte sacrificiel du général romain en pleine bataille pour sauver l'armée, qui pourrait en être le modèle : en effet le général dévoue d'abord les ennemis aux dieux infernaux, puis fait don de sa propre personne, selon tout un rituel qui désigne cet acte comme profondément sacré.

Dans l'Antiquité, les penseurs ont souvent débattu des possibles conflits d'intérêt entre l'amour de la patrie et le souci de la famille ou de l'amitié, ou encore de l'opposition entre la conquête, qui permet d'accroître le patrimoine de la cité, et la justice, qui ordonne de veiller sur le genre humain ${ }^{2}$. Ils se sont plus rarement intéressés à la tension entre le devoir patriotique et le devoir d'humanité. Cicéron fut sans doute le premier et l'un des rares à y réfléchir, suivi en cela par Augustin. Dans le de officiis I, 152 suiv., s'interrogeant sur la nature de l' honestas d'après Panaetius, il la décrit comme composée de quatre éléments : la cognitio (la poursuite du savoir), la communitas (les devoirs envers la communauté), la magnanimitas (la grandeur d'âme, le courage) et la moderatio. Puis discutant la hiérarchie entre ces éléments, il place la communitas au-dessus de la cognitio, mais affirme de manière claire que la communitas ne doit pas entrer en conflit avec la moderatio : certaines actions sont trop viles pour que le sage les accomplisse, dit-il, et même s'il s'agit de sauver sa patrie. Une idée qui lui est sans doute personnelle ${ }^{3}$. C'est aussi lui qui théorise l'humanitas commme concept politique et propose un cosmopolitisme non pas moral (comme celui des Stoïciens) mais politique. Est-il possible toutefois de promettre aux défenseurs armés de la patrie l'éternité dans l'au-delà ${ }^{4}$, et en même temps d'exalter l'humanitas? " Notre patrie a des droits sur nous. Mais comment au mieux remplir ses obligations quand précisément nous sommes attachés à accroître les

aliud bonum est; omnia enim relinquis ut hoc habeas. Vide quanta vis honesti sit : pro re publica morieris, etiam si statim facturus hoc eris cum scieris tibi esse faciendum.)

Ainsi, selon la tradition rapportée par Cicéron, Carnéade affirmait au II $^{\mathrm{e}}$ siècle que toute conquête était injustice et que les Romains devraient, s'ils voulaient être justes, restituer ce qui ne leur appartenait pas (de republica III, 8, 12). "La sagesse (sapientia) nous ordonne d'accroittre notre patrimoine, mais la justice de veiller sur le genre humain (consulere generi hominum : III, 14, 22) et de ne point porter atteinte au bien d'autrui. " Pour les Stoïciens, en revanche, il était possible de mener un empire justement, c'est-àdire humainement : il fallait seulement être cléments avec ceux qu'on avait vaincus. Cf. J.-L. FerRary, Philhellénisme et impérialisme. Aspects idéologiques de la conquête romaine du monde hellénistique, Rome, 1988.

3 S'inspire-t-il de Posidonius ? Cicéron dit dans ce passage (de officiis I, 159) qu'il a entendu parler d'un ouvrage où Posidonius dresse la liste des actions ignominieuses que le sage ne peut accomplir, même au nom de la patrie ; mais il est peu probable qu'il ait eu l'ouvrage entre les mains (cf. I.G. KidD ed, Posidonius, vol. II : The Commentary, 2, Cambridge, 1988 (réimp. 2004) : F. 177, p. 652-53).

4 Cf. de republica VI, 12, 13 : « ceux qui ont servi ou accru leur cité ont une place dans le ciel. " 
biens de l'humanité ?" (de republica I, 2, 3). L'humanitas, la caritas generis humani (de finibus V, 23, 65) peut-elle vraiment être une valeur efficiente ? Ou faut-il penser, avec Rousseau, que notre notion d'homme ne dépasse pas les limites de la patrie ?

Ce conflit entre patriotisme et humanitas n'apparaît que dans ces temps où se pense non pas seulement la citoyenneté du monde, mais la possiblilté d'appartenir de manière efficiente à deux mondes différents : le monde et la patrie locale ${ }^{5}$, ou pour les Chrétiens la patrie terrestre et la patrie céleste. Dans tous les cas il oppose deux valeurs de degré différent, la terre, qui implique un engagement collectif, et le principe, qui suppose avant tout une conscience individuelle. Ce problème se pose pour les Romains avec l'extension de la communauté civique et des conquêtes à la fin de la République ; et il se reposera de manière nouvelle lorsque l'État deviendra chrétien à la fin du $\mathrm{IV}^{\mathrm{e}}$ siècle. C'est sur ces deux moments que nous proposons de nous arrêter, à travers la réflexion cicéronienne et celle d'Augustin. Mais commençons par préciser les deux concepts, celui d'amor patriae et celui d'humanitas.

\section{De l'amour de la patrie à l'amour de la res publica}

L'amour de la patrie est la vertu par excellence du citoyen antique par laquelle l'héroïsme se définit non comme une sortie de la condition humaine, mais comme une part du politique. La tradition, reprise jusqu'à Augustin, veut même que les Romains aient mérité leur dominium à cause de ce "bien divin ". Mais alors que, jusqu'au $\mathrm{I}^{\text {er }}$ siècle, cette vertu concerne toujours la terre-patrie, locale et concrète, qu'elle soit polis ou civitas, l'extension de la citoyenneté à tous les Italiens au Ir siècle avant notre ère fait apparaître un nouvel objet : la caritas rei publicae ${ }^{6}$. Ainsi s'opère un double déplacement : tout d'abord du registre familial, le terme caritas est passé au registre politique et l'on comprend que cette vertu qui est aussi une passion est, pour reprendre une terminologie stoïcienne, un bon sentiment, un sentiment naturel, comme celui que nous éprouvons à l'égard de nos parents ${ }^{7}$; d'autre part d'amour de la terre-patrie, le terme désigne l'affection pour la communis patria, la cité romaine en tant qu'elle

5 Sénèque pense au contraire séparément les deux, le monde (ad Lucilium 3, 28, 4 : patria mea totus hic mundus est; cf. 102, 21 : patria est illi quodcumque suprema et universa circuitu suo cingit, hoc omne convexum intra quod iacent maria cum terris, intra quod aer humanis divina secernens etiam coniungit, in quo disposita tot numina in actus suos excubant) et la patrie locale : ad Lucilium 9, 76, 27 (ci-dessus n. 1).

6 Dans les Partitiones oratoriae 16, 56, Cicéron distingue caritas (deorum, patriae, parentum), amor (fratrum, coniuguum), honestas (virtutum), trois sentiments utiles au bien commun ; cf. Sall., B. Catil. 58 : nos pro patria, pro libertate, pro vita certamus (id., 59) ; cf. Tite-Live II, 2. On trouve aussi bien sûr pietas : de inventione 22, 66 ; Tite-Live VII, 40.

7 Mais plus grand que l'amour des siens : cf. de republica VI, 16, 6: "toi Scipion, pratique la justice et les devoirs ; ils sont considérables quand il s'agit des parents et des proches, mais ils sont les plus grands de tous, quand il s'agit de la patrie ». Pourtant dans ce dialo- 
réunit tous les citoyens où qu'ils soient et leur donne leur citoyenneté (de legibus II, 5). Le passage de l'amor patriae à la caritas rei publicae va ainsi de pair avec l'extension géographique et intellectuelle du concept de res publica ${ }^{8}$. Or l'affection pour cette chose abstraite qu'est le bien public ne va pas de soi. Comment aimer une patrie de droit, comment aimer les lois, les institutions, qui donnent seulement la vie civile aux citoyens ${ }^{9}$ ?

L'évolution de la notion révèle la dimension politique du débat sur la vertu à la fin de la république : il s'agit de faire comprendre aux Italiens que la nouvelle patrie, Rome, était fondée non sur des traditions communes, mais d'abord sur la vertu, liée au respect des lois et source de liberté. C'est en effet elle qui fait la respublica. La série pro legibus, pro libertate, pro patria présente dans les Tusculanes 4, 43 et dans d'autres textes cicéroniens se retrouve chez de nombreux auteurs, jusqu'à Quintilien qui explicite ainsi la distinction devenue rhétorique entre natio et patria ${ }^{10}$ : les coutumes sont le propre des nations (barbare, romaine, grecque), alors que la vertu construit la patria; la coutume met l'accent sur la spécificité de chaque peuple, la vertu mène à l'idée abstraite de bien commun.

Ce moment d'exaltation pour la commune patrie ne durera pas. Sous l'Empire, il apparaîtra de plus en plus, comme le souligne Pline le Jeune dans son Panégyrique, que seul l'empereur possède la vertu et se révèle capable de caritas patriae ou rei publicae ( $\$$ $20)$; les citoyens l'aiment, ont de la caritas pour sa personne (\$ 62) ou lui sont fidèles ; mais leur amor patriae ne concerne que leur patrie locale - c'est ce que confirment les inscriptions ${ }^{11}$.

gue, mais plus encore dans le de officiis, la patrie n'est présentée que comme un des cercles d'appartenance de l'homme.

8 Une extension promise à un grand avenir, puisqu'elle permettra au Moyen Âge de penser le transfert à un royaume de cette émotion si particulière qui concernait jusqu'ici la petite cité de naissance (E. Kantorowicz, Les deux corps du roi, Paris, tr. fr., 1989, p. 181 suiv.) Toutefois la chose ne va pas de soi : par exemple, au Moyen Âge, la notion de patrie-république n'est pas susceptible de compassion ; on a de la loyauté pour le roi mais pas un devoir civique pour les citoyens, comme l'a montré encore Kantorowicz.

9 P. Costa, Civitas. Storia della cittandinanza in Europa, Rome-Bari, 1999, p. 29.

10 Institutiones Oratoriae V, 10, 24-26: natio, nam et gentibus proprii mores sunt nec idem in barbaro, Romano, Graeco probabile est. patria, quia similiter etiam civitatum leges instituta opiniones habent differentiam.

11 P. LE Roux, "L'amor patriae dans les cités sous l'Empire romain ", dans Idéologies et valeurs civiques dans le monde romain. Hommages à C. Lepelley», H. INGLEBERT (éd.), Paris, 2002, p. 143-161. 


\section{Le concept d'humanité}

Parallèlement à cette évolution de l'amor patriae, se met en place le concept d'humanitas. Chez Cicéron, il peut désigner la philanthropia ou la civilisation, mais aussi le respect de l'homme en tant qu'homme, défini comme le principe nécessaire du gouvernement des provinces en temps de paix ${ }^{12}$. Ce sens d' humanitas repose sur deux postulats fondamentaux : d'une part le fait que tous les hommes sont liés par un lien naturel - une idée qui s'exprime dans les formules societas humani generis, ou communio societatis etc... ; qu'il est le même partout, "à Athènes comme à Rome "; ou que "un homme, par cela seul qu'il est homme, ne peut absolument pas être un étranger aux yeux d'un autre homme » (de finibus III, 63). D'autre part, la reconnaissance que ce lien n'est pas seulement un sentiment naturel et un principe moral, mais encore un principe politique : pour Cicéron, qui de ce point de vue differe fondamentalement des philosophes de l'époque hellénistique, la société du genre humain est sans doute un des cercles d'appartenance de l'homme, mais elle est aussi liée à la respublica, définie elle-même comme societas iuris ${ }^{13}$. Ainsi non seulement la societas iuris, la communauté politique, est impensable sans le monde, mais l'idée d' humanitas permet de penser politiquement le lien entre le territoire romain et l'orbis terrarum, entre res publica et res extensa : liée temporellement et spatialement, verticalement et horizontalement au monde, la respublica fait ainsi partie d'une chaîne des nations, qu'elle a pour charge de conserver ${ }^{14}$.

Ce lien entre Rome et le monde a constitué un élément majeur dans l'identité politique de Rome. Mais, on le voit bien, la formulation cicéronienne est tout à fait particulière : dans la tradition impériale, que Virgile a résumée en une célèbre formule (" épargner les vaincus et soumettre les superbes ») et qu'on retrouve dans les textes officiels relatifs à la clémence de l'empereur, l'humanitas est fondée sur l'intérêt du vainqueur. C'est ce qu'il faudra entendre dans la fameuse déclaration d'Auguste : "les vaincus que j'aurais pu tuer, je les ai épargnés ${ }^{15}$ » ou dans un texte du droit du $\mathrm{II}^{\mathrm{e}}$ siècle qui explique ainsi l'origine du terme servus : " les esclaves sont ceux que par humanité le vainqueur a épargnés ${ }^{16}$. » Dans ce contexte, l'humanitas n'est pas un principe moral

12 Ad Quintum fratrem I, 1.

13 Cf. de finibus II, 45.

14 Cf. de officiis II, 8, 27 suiv. Ainsi, par exemple, justice intérieure et justice extérieure sontelles liées. Cela explique pourquoi la respublica peut périr non seulement pour des raisons internes (factions ou tyrannie d'un seul), mais aussi lorsqu'elle pratique l'injustice envers les autres peuples, comme ce fut le cas selon Cicéron à partir de Sylla.

15 Res gestae I, 3 : victorque omnibus veniam petentibus civibus peperci. Externas gentes, quibus tuto ignosci potuit, conservare quam excidere malui. Voir les remarques de P. VeYne, "Humanitas ", in A. Giardina (éd.), L’homme romain, Paris, 1992.

16 Digeste I, 5, 4 = Florentinus 9 Institutiones : Servi ex eo appellati sunt, quod imperatores captivos vendere ac per hoc servare nec occidere solent. 
universel, mais le résultat d'un pragmatisme politique car fondamentalement, par la loi de la guerre, le vainqueur a le droit de vie et de mort sur les vaincus.

Pour Cicéron au contraire, les rapports entre gouvernés et gouvernants sont fondés sur la solidarité, sur la co-responsabilité des hommes. Dans cette perspective, l'homme n'est pas considéré comme individu biologique, mais en tant qu'il est apte à former du lien (vinculum iuris), à préserver des rapports de droit (et de fides) avec les autres dans toutes sortes d'associations (societates), que ce soit à travers le mariage, la société politique, les relations avec les autres peuples... Par suite, ceux qui détruisent ce lien entre tous les hommes - le tyran qui ne respecte pas le droit ou l'homme cruel - se mettent hors de l'humanité. Il en est de même de celui qui méprise les étrangers.

De republica II, 26, 48 : "Comment donner à juste titre le nom d'homme à celui qui refuse d'avoir avec ses concitoyens, comme avec l'ensemble du genre humain, toute communauté juridique, toute solidarité humaine?»"

Cette façon de penser le genre humain comme englobé dans un système contractuel qui en reconnaît l'unité naturelle explique enfin que la patria communis, la patrie romaine, qui réunit tous les citoyens romains de quelque origine qu'ils soient, et qui inclut en elle les petites patries de chacun, est la plus chère de toutes (carissima), car elle est celle qui réalise au plus haut point l'humanité de l'homme et la fin de la nature de l'homme. Ainsi les intérêts des deux patries ne peuvent-ils s'opposer. En revanche on voit bien comment l'humanitas peut entrer en contradiction avec la caritas rei publicae. Mais dès lors, quel choix opérer?

\section{La résolution du conflit}

Cette question, Augustin l'aborde de son côté au livre I du traité de libero arbitrio, où l'influence de Cicéron est particulièrement importante ${ }^{17}$ et où il s'interroge sur l'origine du mal, dont il dira ensuite que c'est le libre arbitre (I, 2, 4; 7, 16 suiv.). Dialoguant avec son disciple Evodus, il en arrive à se demander "si pour sauver la vie, la liberté ou l'honneur, on peut tuer sans mauvaise passion (sine ulla libidine) soit un ennemi qui attaque, soit un sicaire qui tend un piège " (I, 5,11$)$. Evodus répond que si les lois ordonnent de tuer l'ennemi, la loi divine, elle, défend de tacher ses mains de sang. Le dialogue propose deux solutions à ce conflit. Pour les sages, le seul critère est la bonne volonté (bona voluntas), par laquelle on désire recte et honeste vivere et parvenir à la summa sapientia (I, 12, 25); par suite, le sage vivra selon la caritas, entendue ici comme pur amour de Dieu et de la patrie céleste, amour de la loi éternelle, cet amour qui nous empêche de tuer un homme $(15,31)$. L'accès à la vie tranquille et bonne, à la vie morale ne peut donc en aucun cas s'accommoder de l'homicide. Mais pour

17 Voir l'édition commentée de F. DE Capitani, Il de libero arbitrio di Sant'Agostino, Commentario e traduzione, Milan, 1987. 
les hommes inexpérimentés (imperiti), la loi terrestre (et changeante, temporalis, \$14) compte le plus et peut supporter un mal moindre - tuer un ennemi menaçant : si la guerre est déclarée par l'autorité de l'État, c'est qu'elle est entreprise pour rétablir la justice $(\$ 13)$. Dans ce cas, et c'est Evodus qui parle, on peut donc choisir de défendre l'intérêt de la patrie.

Trois précisions sont importantes : d'abord c'est un choix, non une obligation absolue ; puis il faut que le dirigeant soit lui-même sans passion et non un tyran cupide; enfin ce qui justifie cette soumission à l'ordre, ce n'est pas seulement la raison d'État, c'est la bonne volonté : le soldat peut tuer à la guerre parce que, obéissant à la loi (minister legis), il se bat pour une cause désintéressée (et non pour s'enrichir par exemple), " il accomplit donc son devoir facilement sans libido, sans mala cupiditas, c'est-à-dire sans mauvaise passion (quare officium suum facile nulla libidine implevit). " Ainsi, dans un moment où l'Empire est chrétien, c'est-à-dire où semblent se réconcilier l'Église et l'État, il est pensable que le pouvoir, à condition qu'il soit légitime, prenne la responsabilité morale de l'homicide. La loi de Dieu " tu ne tueras pas " ne s'applique donc pas aux milites qui choisissent d'accepter les ordres justes du pouvoir légitime ${ }^{18}$ : "On peut donc obéir sans passion (sine libidine) à cette loi qui, pour la défense des citoyens, commande qu'on rejette avec la même violence une violence ennemie (vim hostilem) et on peut le dire de tous les exécutants qui ont été soumis à une autorité par la loi ou par quelque condition sociale (qui iure atque ordine potestatibus quibusque subiecti sunt $)^{19}$." Tous sont en quelque sorte mus par une autre caritas, l'amour de la patrie terrestre, qui est une bonne passion, c'est-à-dire aussi une manière de se transcender. Augustin construit donc une chaîne sémantique : caritas, bona cupiditas, utilitas publica - qui, pour le commun des mortels, peut justifier le meurtre d'un homme. On a là l'embryon de la définition de la souveraineté dans la tradition occidentale comme ce qui décide du légal et du non-légal, et de la loi comme critère de la moralité, de la bonne passion ou du courage honorable ${ }^{20}$. Avec toutefois l'idée que l'homme a toujours, fondamentalement, le choix de ne pas obéir.

18 De libero arbitrio : I, 5, 12 ; cf. J. Flori, La guerre sainte. Formation de l'idée de croisade dans l'Occident chrétien, Paris, 2001.

19 I, 5, 12. N'oublions pas qu'à Rome, obéir à la loi, c'est aussi respecter le serment, prêté au moment de l'enrôlement, de ne pas fuir du champ de bataille sous peine de mort. Polybe (VI, 52, 2) évoquait la colère (orgè) des soldats au combat et la peur du châtiment. Sur ce sujet, voir les remarques de A. BARZANO, "Libenter cupit commori qui sine dubio scit se esse moriturum : la morte per la patria in Roma repubblicana ", in M. Sordi (éd.), Dulce et decorum est pro patria mori, 1990, p. 157-170.

20 Le courage patriotique, c'est la (bonne) passion de l'homme libre, de celui précisément qui est capable de risquer sa vie pour la liberté de tous (à la différence de l'esclave qui ne pense qu'à sa survie biologique). Mais le courage ne suffit pas à lui seul à désigner le bon patriote : le gladiateur aussi est courageux, le tyran aussi peut l'être, mais ils le sont sous l'influence de mauvaises passions, la colère, la cupidité ou la libido dominandi. Il faut donc aussi la caritas pour faire du courage un bon sentiment. De fait, la distinction entre les 
L'argumentation d'Augustin n'est pas nouvelle. Elle s'appuie tout d'abord sur une longue tradition juridique, celle, par exemple, qui débattait de la responsabilité pénale des magistrats. Discutant cette question, le juriste Paul avait expliqué au $\mathrm{III}^{\mathrm{e}}$ siècle qu'un magistrat peut bien agir contre le droit (iniuria) s'il le fait rei publicae venerandae causa $^{21}$; car alors il a agi conformémement à la morale, recte, c'est-à-dire sans intérêt pour lui-même. Ulpien distinguait aussi la violence (vis) atrox et celle quam recte intu$\mathrm{lit}^{22}$ : la différence résidait selon lui dans la motivation, définie selon un double critère, objectif (l'intérêt public) et subjectif (l'intention, l'animus, du magistrat). Pour ces juristes, donc, il était évident que si un magistrat avait commis une injustice par intérêt, il avait agi adversus bonos mores et était coupable, même s'il avait utilisé ses pouvoirs légalement; mais s'il avait agi par utilité publique ou dans l'intention de défendre le prestige de sa fonction ou de la res publica, c'est-à-dire selon une volonté bonne, ses actes étaient considérés comme accomplis recte, de manière droite. La distinction entre le droit (légal/illégal) et la moralité (adversos bonos mores/recte) avait donc deux effets contrastés : elle permettait tantôt d'amender un acte illégal par la prise en compte de l'intérêt public, qui est une bonne passion, tantôt de contrôler l'usage qu'un magistrat faisait de son pouvoir et de son droit $^{23}$.

différentes formes de courage n'est pas seulement un exercice d'école ; elle sert à penser l'ambiguité du patriotisme, ou plutôt la limite étroite entre le rebelle et le patriote, l'assassin et le tyrannicide, c'est-à-dire entre la passion, la libido, la mala cupiditas, et la vertu. À plusieurs reprises, on se posera la question et notamment à la mort de César : les assassins du tyran étaient-ils des libérateurs ou des parricides ?

21 Paul, 10 ad Sabinum = Digeste 47.10.33 : Quod rei publicae venerandae causa secundum bonos mores fit, etiamsi ad contumeliam alicuius pertinet, quia tamen non ea mente magistratus facit, ut iniuriam faciat, sed ad vindictam maiestatis publicae respiciat, actione iniuriarum non tenetur. "Ce qu'un magistrat a fait selon les bonnes mœurs par dévouement pour la res publica, même s'il en est résulté une injustice faite à autrui, dans la mesure où il l'a fait sans intention de commettre une injustice, mais pour revendiquer la majesté de l'État, ne peut être l'objet d'une action pour iniuria. »Cf. aussi : Digeste 47, 10, 15, 39 (Ulpien, 77 ad edictum). Unde quaerit Labeo, si magistratus municipalis servum meum loris ruperit, an possim cum eo experiri, quasi adversus bonos mores verberaverit. Et ait iudicem debere inquirere, quid facientem servum meum verberaverit : nam si honorem ornamentaque petulanter adtemptantem ceciderit, absolvendum eum. "Si un magistrat municipal a blessé mon esclave avec un fouet, Labeo demande si je peux intenter une action à ce magistrat pour avoir frappé mon esclave contre les bonnes mœurs. Il dit que le juge doit chercher à savoir ce qu'a fait l'esclave pour être frappé ; car si le magistrat l'a pris en train d'attenter à sa charge ou à ses insignes, alors il doit être absous. "

22 Ulpien 11 ad edictum = Digeste 4.2.3.1 : Sed vim accipimus atrocem et eam, quae adversus bonos mores fiat, non eam quam magistratus recte intulit; scilicet, iure licito et iure honoris quem sustinet. "Nous considérons comme atrox la violence commise contre les bonnes mœurs, non celle qu'un magistrat a commise recte; car alors c'est en vertu du droit et des pouvoirs de sa charge qu'il l'a commise."

23 Un amendement à la formulation d'Ulpien (57 ad edictum = Digeste 47.10.13.6) selon laquelle «ce qu'un magistrat a fait en vertu des pouvoirs de sa fonction, ne peut donner 


\section{Les paradoxes du patriotisme}

C'est aussi autour de ces notions qu'une partie du conflit politique s'était cristallisée à la fin de la république, après l'assassinat de Tibérius et après chaque recours au senatus-consulte dit ultime ${ }^{24}$. Cicéron défendait lui-même cette distinction entre le légal et le moralement juste dans le Songe de Scipion et soutenait qu'on peut tuer un citoyen s'il menace la res publica ${ }^{25}$. En revanche, sa position était moins tranchée quelques années plus tard, dans le de officiis, où il reconnaissait qu'il était des choses que le bon citoyen ne pouvait faire pour la patrie car il y a une loi suprême, supérieure à celle de la patrie, qui pousse à respecter l'homme ${ }^{26}$. De même, après la mort de César, il invoquait la responsabilité de l'individu, qu'il fût sage ou non, que ses choix fussent inspirés par l'amitié, l'ordre à l'autorité ou l'intérêt de l'État. Par exemple, écrivait-il dans ses Lettres, ceux qui, tel Caius Matius, avaient obéi à César ne pouvaient se cacher derrière l'obéissance aveugle au tyran ou l'amitié pour justifier leur soutien : c'est par choix qu'ils avaient obéi, ils étaient donc directement responsables ${ }^{27}$. Ainsi, avant Augustin, ne prônait-il ni l'amour aveugle pour la patrie ni l'amour absolu de l'humanité mais l'examen de conscience individuel, seul principe d'action.

Dans les dernières années de sa vie, Cicéron semble avoir donc renoncé, théoriquement du moins, au principe d'utilité publique comme critère de la bonne action, pour suggérer que la justice est le critère de la bonne patrie (et du bon dirigeant) : une bonne patrie ne peut exiger le pire de l'homme. N'est-ce pas aussi ce qu'il exigeait du bon gouvernant ou d'un ami digne d'être aimé ? Tel serait l'un des paradoxes du patriotisme qu'on ne peut défendre la patrie que si elle exige des actes moraux. Manière aussi de justifier la résistance voire la mort du tyran.

lieu à une action pour iniuria" (quae iure potestatis a magistratu fiunt, ad iniuriarum actionem non pertinent). Voir le commentaire de O. LICANDRO, In magistratu damnari. Ricerche sulla responsabilità dei magistrati romani durante l'esercizio delle funzioni, Turin, 1999 , p. 48 suiv.

24 Voir A. Lintott, Violence in Republican Rome, 1968, 1999, p. 31 suiv. et C. Moatti, "Des choses publiques à la chose du peuple », in C. Moatti et M. Riot SARCey (éd.), La République dans tous ses états, Paris, 2009, p. 258 suiv.

25 Le détail de ces textes se trouve dans C. MoatTi, "Des choses publiques à la chose du peuple " et EAD., "Conservare rem publicam ", à paraître dans Études philosophiques, 2011.

26 De officiis I, 45, 159; I, 11, 34.

27 Cicéron, de amicitia 11, 39 suiv. ; Philippiques II, 11, 27 : Trébonius a tué César, préférant la République à l'amitié ; et de fait, ajoute Cicéron, la seule bonne amitié est celle qui ne met pas en cause les intérêts de l'État. Sur les lettres, voir aussi C. MoAtTi, La raison de Rome, Paris, 1997, p. 209-210. 


\section{L’inhumanité du tyran}

L'exception que constitue le cas du tyran est un lieu commun de la pensée politique antique. Mais la comparaison entre Augustin et Cicéron est éclairante. Pour le premier l'obéissance à la loi qui prescrit l'homicide ne peut se justifier, nous l'avons dit, que si elle émane d'un législateur doté de bonne volonté et expers omnino libidinis, non si elle est le fait d'un homme corrompu, qui, par son éloquence, aurait obtenu un pouvoir tyrannique (qui tyrannicam potestatem nanctus) (I, 5, 12). Cicéron va plus loin : le tyran n'est pas seulement un individu dépouillé de bonne volonté, c'est un homme qui s'est placé hors de l'humanité. Pour penser la tyrannie et justifier le tyrannicide, il invite à penser l'inhumanité du tyran, c'est-à-dire à la fois l'inhumanitas (qui signifie le fait de s'abandonner aux passions déchaînées et mauvaises) et l'immanitas qui est l'équivalent de la cruauté et de la déloyauté (Philippiques II, 4,7). C'est parce qu'il est inhumain que le tyran peut être tué violemment et, de même, parce qu'il s'est mis hors de la société que le séditieux peut être déclaré homo sacer, parce qu'il a été cruel que l'ennemi doit être exterminé sans merci. Leur mort ne menace pas la chaîne de l'humanité ou de la société politique, puisqu'ils se sont mis au ban de la société humaine.

Toutefois tuer les séditieux, exterminer les ennemis et assassiner un tyran, n'est-ce pas courir le risque de se mettre soi-même hors de l'humain ? Être cruel envers les cruels, n'est-ce pas le fait d'un homme soumis aux passions? C'est ce que Salluste fait dire à César dans son Catilina lorsqu'il évoque le débat au Sénat en 63 sur le sort des conjurés. Contre Silanus, auteur de la proposition concernant la peine de mort, contre Caton et Cicéron, qui le soutiennent, César plaidait en faveur de la prison, et pour deux raisons. D'abord par respect des lois romaines qui interdisent de mettre à mort un citoyen sans procès, si bien que le légalisme se mettait au service de l'humanitas: la proposition de Silanus lui paraissait en effet « non pas cruelle (cruedelis) [...] mais contraire à la res publica" (aliena a re publica nostra) (Cat. 51); l'autre raison invoquée était la dignité des sénateurs, c'est-à-dire leur moralité : refuser d'agir par colère (iracundia) ou par peur (metus), de se laisser dominer par des mauvaises passions permettait de ne pas entrer dans un processus de violence. La moralité était même un argument supérieur à la légalité. On retrouve ici la distinction entre agir légalement (iure) et agir moralement (recte), qui tantôt s'opposent, tantôt se renforcent l'un l'autre.

La question que soulève César dans le texte de Salluste revient sans cesse dans le débat politique de la fin de la république. Elle se pose par exemple après les Ides de mars : la mort du tyran met-elle fin à la tyrannie ? quel sort doit-on réserver aux partisans d'un ennemi public ? Cicéron, qui en 63 avait fait mettre à mort les partisans de Catilina, demanda à Brutus la mort des amis d'Antoine après le décret sénatorial qui avait désigné ce dernier comme hostis : "Soyons cléments et nous perpétuerons les guerres civiles ", expliquait-il ${ }^{28}$. Brutus refusa net, en l'accusant de se laisser aller à 
l'iracundia et en prônant la moderatio, cette vertu dont Cicéron avait fait l'éloge dans le de officiiis ${ }^{29}$ : quelques mois plus tard, Cicéron lui-même subira le sort qu'il avait réclamé pour ses adversaires politiques.

Est-il donc possible de mettre un terme au cycle sans fin de l'inhumanité ? Certains historiens antiques donnèrent une réponse claire : l'état d'exception et de violence que font naitre la tyrannie et la mort du tyran ne prend fin que par la mort violente du tyrannicide lui-même, plus précisément par son suicide. C'est ainsi que s'expliquait la mort de Brutus et Cassius : pour que l'ordre revienne, les héros sont condamnés à se sacrifier ${ }^{30}$.

Tuer pour la patrie ne pouvait ainsi s'achever que dans la mort pour la patrie. Le mori pro patria est une expression elliptique qui occulte la face la moins glorieuse du patriotisme. Il faut la rigidité du rituel religieux (la devotio) pour le reconnaitre, ou les détours de la pensée utopique (penser le sage comme un homme sans passion et donc incapable d'homicide) pour en déconstruire l'évidence, mais l'inhumanité de la patrie reste le plus souvent un impensé du politique. C’est seulement dans les périodes de grande tyrannie que l'homme lucide peut dénoncer les sanglantes aspirations de sa patrie et la quitter pour une autre. Qu'est-ce en effet qu'une patrie ? Non pas le lieu où ma famille est née, comme le pensent la plupart des hommes; ni le lieu où je me sens bien, comme disaient certains Stoiciens ; mais, comme le comprennent Cicéron et Augustin, le lieu que je choisis en fonction de mes valeurs ${ }^{31}$.

Claudia MолтT
Professeur à l'Université Paris 8
et à l'University of Southern California
moatti@usc.edu

29 Ad Brutum 11, 2 ; cf. aussi les remarques de LinTotT, Violence in Republican Rome, p. 48.

30 Plutarque, Brutus 54 (1), 6 ; mais surtout Appien, Guerres civiles IV, 134.

31 À cette question, le traité de Favorinus d'Arles, sur l'exil, apporte des réponses tout à fait importantes. Cf. C. MоAтTI, « De la peregrinatio comme stratégie intellectuelle dans l'Empire romain au II siècle de notre ère ", MEFRIM 119, 1 (2007), p. 129-136. 